\title{
Direction Tracking of Multiple Moving Targets Using Quantum Particle Swarm Optimization
}

\author{
Hongyuan Gao , Jia Li and Yanan Du \\ College of Information and Communication Engineering, Harbin Engineering University, Harbin, China
}

\begin{abstract}
Based on weighted signal covariance (WSC) matrix and maximum likelihood (ML) estimation, a directionof-arrival (DOA) estimation method of multiple moving targets is designed and named as WSC-ML in the presence of impulse noise. In order to overcome the shortcoming of the multidimensional search cost of maximum likelihood estimation, a novel continuous quantum particle swarm optimization (QPSO) is proposed for this continuous optimization problem. And a tracking method of multiple moving targets in impulsive noise environment is proposed and named as QPSO-WSC-ML. Later, we make use of rank-one updating to update the weighted signal covariance matrix of WSC-ML. Simulation results illustrate the proposed QPSO-WSC-ML method is efficient and robust for the direction tracking of multiple moving targets in the presence of impulse noise.
\end{abstract}

\section{Introduction}

In array signal processing domain, the estimation of DOA has been a top research subject in the past several decades, and the technology of DOA estimation is of great interest for many sensor systems such as radar, sonar, electronic surveillance, seismic exploration and so on [1]. DOA estimation makes use of the signal received by the sensor array to estimate the direction of the target, hence, the algorithm of estimation directly affects its performance. Numerous improved methods have been proposed after conventional methods, such as the maximum likelihood (ML) estimation and approximate maximum likelihood estimation [2-3]. In the spatial spectrum estimation, the method is simple and has excellent performances in both asymptotic and threshold domains. Nevertheless, the process of implementation is complex, which involves a multidimensional nonlinear optimization problem with an increased computational burden. With the rapid development of intelligent computing technology, scholars began to estimate the directions of arrival by all kinds of intelligent algorithms, which have undoubtedly been powerful tools to overcome the drawback of the method [4-6].

As conventional DOA estimation methods assumed that the noise environment is Gaussian noise, however, in the practice, many random signals and noises are not Gaussian distribution, such as lightning atmospheric noises, the underwater acoustic signals, the line instantaneous peak voice signals of the communications as well as a lot of man-made noises. Above noises have a notable peak value characteristic, and may be described by different characteristics of the $\mathrm{S} \alpha \mathrm{S}$ process in which second order moment and high order moment do not exist, so some low order moments are designed to solve this problem [7-8]. At present, dynamic tracking is studied in the Gaussian noise environment, but dynamic tracking of the impulse noise environment is still a theory difficulty.

Therefore, in order to solve dynamic tracking problem of the impulse noise environment, a WSC-ML method is proposed, then the QPSO algorithm is designed to solve tracking equation of the WSC-ML method under impulsive noise environment. The proposed tracking method of multiple moving target is called as QPSOWSC-ML. This method can avoid the decompositions of the fractional lower order moments matrix which should be repeated in the method based on subspace tracking, and performs better than other methods based on subspace tracking in the aspect of tracking precision.

\section{Tracking model of multiple moving targets}

Consider a uniform linear array consisting of $N$ identical omni-directional antennas, $M$ narrowband sources with wavelength $\lambda$ impinge on the array from $\left\{\theta_{1}, \theta_{2}, \ldots, \theta_{M}\right\}$. Assume that the distance between neighborhood antennas is $\lambda / 2$. The $k$ th snapshot sample data which is received by linear array is defined as

$$
\boldsymbol{x}(k)=\boldsymbol{A}(\boldsymbol{\theta}) \boldsymbol{s}(k)+\boldsymbol{n}(k),
$$

where $\boldsymbol{x}(k)$ is the $N \times 1$ vector of the signals received by the array sensors, $\boldsymbol{s}(k)$ denotes the $M \times 1$ signal vector, $\boldsymbol{A}(\theta)=\left[\boldsymbol{a}\left(\theta_{1}\right), \boldsymbol{a}\left(\theta_{2}\right), \ldots, \boldsymbol{a}\left(\theta_{M}\right)\right]$ represents steering matrix, $\boldsymbol{n}(k)$ denotes the $N \times 1$ complex $\mathrm{S} \alpha \mathrm{S}$ distribution noise 
vector. The $m$ th steering vector toward direction $\theta_{m}$ is written as $\boldsymbol{a}_{m}=\left[1, \mathrm{e}^{-\mathrm{j} 2 \pi d_{2} \sin \left(\theta_{m}\right) / \lambda}, \ldots, \mathrm{e}^{-\mathrm{j} 2 \pi d_{N} \sin \left(\theta_{m}\right) / \lambda}\right]^{\mathrm{T}}$ $(m=1,2, \ldots, M)$, where $d_{i}(i=2, \ldots, N)$ represents the distance between the $i$ th sensor and the first sensor.

Since second order moment and high order moment do not exist in impulse noise environment, we define weighted received signal vector as $\overline{\boldsymbol{x}}(k)=\left[\bar{x}_{1}(k), \bar{x}_{2}(k), \ldots, \bar{x}_{N}(k)\right]^{\mathrm{T}}, \overline{\boldsymbol{x}}(k)=\boldsymbol{x}(k) /\left(\max _{1 \leq i \leq N}\left|x_{i}(k)\right|^{\sigma}\right)$, where $\sigma$ represents a constant. The weighted signal covariance (WSC) matrix of the first snapshot sampling data can be estimated by the following equation.

$$
C(1)=\bar{x}(1) \bar{x}^{\mathrm{H}}(1)
$$

For the $(k+1)$ th snapshot sample data, the weighted signal covariance matrix is updated as follows.

$$
\boldsymbol{C}(k+1)=\mu \boldsymbol{C}(k)+(1-\mu) \overline{\boldsymbol{C}}(k+1)
$$

where $\overline{\boldsymbol{C}}(k+1)=\overline{\boldsymbol{x}}(k+1) \overline{\boldsymbol{x}}^{\mathrm{H}}(k+1)$ is the covariance matrix of the $(k+1)$ th weighted snapshot sample data, and $\boldsymbol{C}(k)$ is weighted signal covariance matrix of the $k$ th received data, $\mu$ denotes the updating factor.

The tracking problem is to estimate the angle $\theta_{m}(m=1,2, \ldots, M)$ of the sources from $k$ samples ("snapshots") of the received signals. The maximum likelihood estimation of the sources named WSC-ML is described as

$$
\overline{\boldsymbol{\theta}}=\arg \max _{\boldsymbol{\theta}}\left[\operatorname{trace}\left(\boldsymbol{P}_{A(\boldsymbol{\theta})} \boldsymbol{C}(k)\right)\right]
$$

where trace( ) represents the trace function of the matrix, $\boldsymbol{P}_{\boldsymbol{A}(\boldsymbol{\theta})}=\boldsymbol{A}(\boldsymbol{\theta})\left(\boldsymbol{A}^{\mathrm{H}}(\boldsymbol{\theta}) \boldsymbol{A}(\boldsymbol{\theta})\right)^{-1} \boldsymbol{A}^{\mathrm{H}}(\boldsymbol{\theta}) \quad$ represents the projection matrix of steering matrix $\boldsymbol{A}(\boldsymbol{\theta}), \boldsymbol{C}(k)$ is weighted signal covariance matrix of the $k$ th sampling data.

The DOA estimation of the proposed WSC-ML could be considered as a classical continuous optimization algorithm, hence, we use the proposed continuous QPSO algorithm as the optimization method to search for the global optimal solution of WSC-ML and find the directions of moving targets.

\section{Direction tracking of multiple moving targets based on QPSO}

\subsection{Quantum particle swarm optimization}

Quantum particle swarm optimization is a novel intelligent optimization algorithm inspired by social and quantum behavior metaphor of particles. In the proposed QPSO algorithm, a quantum position can be used to produce the corresponding particle's position which represents a latent solution. The quantum position of the $q$ th particle is defined as

$$
\begin{aligned}
\mathbf{y}_{q} & =\left[\begin{array}{llll}
\mathbf{y}_{q 1} & \mathbf{y}_{q 2} & \ldots & \mathbf{y}_{q M}
\end{array}\right] \\
& =\left[\begin{array}{cccc}
\alpha_{q 1} & \alpha_{q 2} & \ldots & \alpha_{q M} \\
\beta_{q 1} & \beta_{q 2} & \ldots & \beta_{q M}
\end{array}\right],
\end{aligned}
$$

where $\alpha_{q m}$ and $\beta_{q m}$ represent a pair of quantum bits of the $m$ th $(m=1,2, \ldots, M)$ quantum bit position of the $q$ th quantum position. Additionally, $\left|\alpha_{q m}\right|^{2}+\left|\beta_{q m}\right|^{2}=1$, $0 \leq \alpha_{q m} \leq 1$ and $0 \leq \beta_{q m} \leq 1$.

There are $Q$ particles in the space of $M$ dimensions in the quantum particle swarm. $\mathbf{y}_{q}=\left[\mathbf{y}_{q 1}, \mathbf{y}_{q 2}, \ldots, \mathbf{y}_{q M}\right]$ $(q=1,2, \ldots, Q)$ denotes the quantum position of the $q$ th particle. $\mathbf{v}_{q}=\left[v_{q 1}, v_{q 2}, \ldots, v_{q M}\right], \mathbf{p}_{q}=\left[p_{q 1}, p_{q 2}, \ldots, p_{q M}\right]$ and $\mathbf{p}_{g}=\left[p_{g 1}, p_{g 2}, \ldots, p_{g M}\right]$ denote the velocity of the $q$ th $(q=1,2, \ldots, Q)$ particle, the best quantum position (e.g. the local optimal quantum position) of the $q$ th particle and the best quantum position (e.g. the global optimal quantum position) of the whole quantum particle swarm, respectively. Therefore, the velocity of the $q$ th particle is updated as

$$
v_{q m}^{t+1}=w v_{q m}^{t}+c_{1} r_{1}\left(p_{q m}^{t}-\beta_{q m}^{t}\right)+c_{2} r_{2}\left(p_{g m}^{t}-\beta_{q m}^{t}\right),
$$

where $c_{1}$ and $c_{2}$ are the acceleration coefficients that control the maximum step length of flying to both the local optimal quantum position and the whole optimal quantum position, $r_{1}$ and $r_{2}$ represent the uniform random numbers between 0 and 1 . Additionally, $w$ denotes the weighted coefficient between 0.4 and 0.9 , which is expressed as

$$
w=w_{\max }-t \times\left(w_{\max }-w_{\min }\right) / t_{\max },
$$

where $w_{\max }, w_{\min }, t$ and $t_{\max }$ denote the maximum weighted coefficient, the minimum weighted coefficient, the current number of iterations and the maximum number of iterations, respectively.

If the particle's velocity $v_{q m}^{t+1}$ exceeds the boundary of upper bound and lower bound, $v_{q m}^{t+1}$ will be subject to

$$
v_{q m}^{t+1}=\left\{\begin{array}{c}
v_{m, \text { max }}, v_{q m}^{t+1}>v_{m, \text { max }} \\
-v_{m, \text { max }}, v_{q m}^{t+1}<-v_{m, \text { max }},
\end{array}\right.
$$

where $v_{m \text {,max }}$ represents the maximum boundary of the $m$ th dimension of velocity.

The evolutionary process of quantum position is mainly completed through quantum rotation gate [9-10]. In the algorithm, the $m$ th dimension of quantum position of the $q$ th particle $\boldsymbol{y}_{q m}$ is updated as 


$$
\begin{aligned}
& \boldsymbol{y}_{q m}^{t+1}=\operatorname{abs}\left[\boldsymbol{U}\left(v_{q m}^{t+1}\right) \boldsymbol{y}_{q m}^{t}\right] \\
& =\operatorname{abs}\left(\left[\begin{array}{cc}
\cos \left(v_{q m}^{t+1}\right) & -\sin \left(v_{q m}^{t+1}\right) \\
\sin \left(v_{q m}^{t+1}\right) & \cos \left(v_{q m}^{t+1}\right)
\end{array}\right] \boldsymbol{y}_{q m}^{t}\right),
\end{aligned}
$$

where abs( ) represents the absolute value function which restrains quantum bit in the range of $[0,1]$, and $v_{q m}$ denotes the $m$ th dimension of velocity of the $q$ th particle. Additionally, $t$ represents the number of iterations of QPSO.

\subsection{Direction tracking of multiple moving targets based on QPSO}

In QPSO, the initial searching bound of the first snapshot is limited to defined domain of direction. As for the other snapshots, the searching space will change with the past searching value and the related parameter when the signal sources move. And the searching bound is derived as

$$
\left\{\begin{array}{l}
z_{m}^{\min }(k+1)=z_{m}^{c}(k+1)-\varepsilon^{k}\left|z_{m}^{\min }(k)-\bar{z}_{m}(k)\right|-r \\
z_{m}^{\max }(k+1)=z_{m}^{c}(k+1)+\varepsilon^{k}\left|z_{m}^{\max }(k)-\bar{z}_{m}(k)\right|+r
\end{array}\right.
$$

where $\varepsilon$ denotes the convergence factor which affects the rate of convergence of the searching space, $r$ represents the searching radius of searching space, $z_{m}^{\min }(k)$ represents the $m$ th-dimension of searching lower bound of the $k$ th snapshot within searching space, $z_{m}^{\max }(k)$ represents the $m$ th-dimension of searching upper bound of the $k$ th snapshot within searching space, $\bar{z}_{m}(k)$ represents the $m$ th-dimension of estimated value of the $k$ th snapshot and $z_{m}^{c}(k+1)$ represents the central value of the $(k+1)$ th snapshot within searching space. Moreover, $z_{m}^{c}(k+1)$ is written as

$$
z_{m}^{c}(k+1)=\delta z_{m}^{c}(k)+(1-\delta) \bar{z}_{m}(k),
$$

where $\delta$ denotes the genetic factor.

Suppose that $\mathbf{z}_{q}=\left[z_{q 1}, z_{q 2}, \ldots, z_{q M}\right]$ represents the $q$ th particle's position which is the corresponding mapping state of quantum position $\overline{\mathbf{y}}_{q}=\left[\beta_{q 1}, \beta_{q 2}, \ldots, \beta_{q M}\right]$, where $0 \leq \beta_{q m} \leq 1(m=1,2, \ldots, M)$. The $m$ th dimension of position of the $q$ th particle is obtained by $z_{q m}=z_{m}^{\min }+\beta_{q m}\left(z_{m}^{\max }-z_{m}^{\min }\right), \quad z_{q m} \in\left[z_{m}^{\min }, z_{m}^{\max }\right]$, where $z_{m}^{\min }$ and $z_{m}^{\max }$ represent the lower bound and upper bound of the $m$ th dimension of variable, respectively.

The initial population of QPSO is created randomly within the quantum domain and the fitness function is used to evaluate the status of each particle. For the proposed tracking method, the fitness of the $q$ th particle's quantum position is calculated as follows.

$$
f\left(\boldsymbol{z}_{q}\right)=\operatorname{trace}\left[\boldsymbol{P}_{A\left(z_{q}\right)} \boldsymbol{C}(k)\right]
$$

According to introduction and analysis above, multiple targets tracking method of QPSO-WSC-ML is described as follows.

Step 1: According to the first snapshot sample data, initialize weighted signal covariance matrix $\boldsymbol{C}(1)$, where $\boldsymbol{C}(1)=\overline{\boldsymbol{x}}(1) \overline{\boldsymbol{x}}^{\mathrm{H}}(1)$.

Step 2: Initialize searching boundary with definition domain of variants, and initialize quantum position and velocity of quantum particle swarm by random method.

Step 3: Compute the fitness of the quantum position of each particle by (12), and initialize the local optimal quantum position and the global optimal quantum position.

Step 4: Number of iterations for direction finding of the $k$ th snapshot is set as $t_{\max }=H \cdot \operatorname{round}\left[z_{1}^{\max }(k)-z_{1}^{\min }(k)\right]$, where $H$ is set as constant and round( ) represents rounding function, and update the velocity and the quantum position of each particle.

Step 5: According to the corresponding position of the quantum position, compute corresponding fitness of the quantum position.

Step 6: Update the local optimal quantum position of each particle and the global optimal quantum position of the whole quantum particle swarm.

Step 7: If the end condition is not met (the end condition is set as maximum number of iterations $t_{\max }$ ), then $t=t+1$, and back to Step 4; else output the global optimal position and go to next step.

Step 8: Update weighted signal covariance matrix by $\boldsymbol{C}(k+1)=\mu \boldsymbol{C}(k)+(1-\mu) \overline{\boldsymbol{C}}(k+1)$ and the searching bound is updated by (10).

Step 9: If $k \geq k_{\max }$, where $k_{\max }$ represents the maximum number of sampling, end the loop; else $k=k+1$ and go to Step 2 .

\section{Simulation results and evaluation}

In this section, we will give the simulation of the proposed QPSO-WSC-ML algorithms in impulsive noise background via computer simulations. Consider that direction tracking system is equipped with uniform linear arrays with half wavelength space, and the number of antenna is set as 8 . The maximum number of sampling $k_{\max }$ is set as 500 , and the impulsive noise is assumed to follow the standard $\mathrm{SaS}$ distribution with the characteristic exponent $\alpha$. Generalized Signal-to-Noise Ratio (GSNR) is computed by $G S N R=10 \lg \left[E\left(|S(k)|^{2}\right) / \gamma\right]$, where $E\left(|s(k)|^{2}\right)$ represents the signals' average power and $\gamma$ represents the dispersion of impulse noise.

In the following, in order to illustrate the superiority of the proposed QPSO-WSC-ML, we compare QPSOWSC-ML with PSO-FLOM-ML which was proposed in [11]. For QPSO, some parameters are set as follows, 
$\varepsilon=0.995, \mu=0.95, r=3, \delta=0.8, H=6, \sigma=0.7$, $w_{\max }=0.9, w_{\min }=0.4, v_{m, \text { max }}=0.2, c_{1}=c_{2}=1$. For PSO and QPSO, the size of population is set as 20 .

Two independent signal sources coming from $\theta_{1}(k)=[-12-0.01 k]^{\circ}$ and $\theta_{2}(k)=[28+0.01 k]^{\circ}$ are considered. For two independent signal sources, Fig. 1 and Fig. 2 depict the direction tracking performance of the two tracking algorithms with $\alpha=1.85$ and GSNR $=4 \mathrm{~dB}$. Moreover, Fig. 1 and Fig. 2 show that QPSO-WSC-ML is more robust than PSO-FLOM-ML.



Figure 1. Tacking of two independent signal sources based on QPSO-WSC-ML

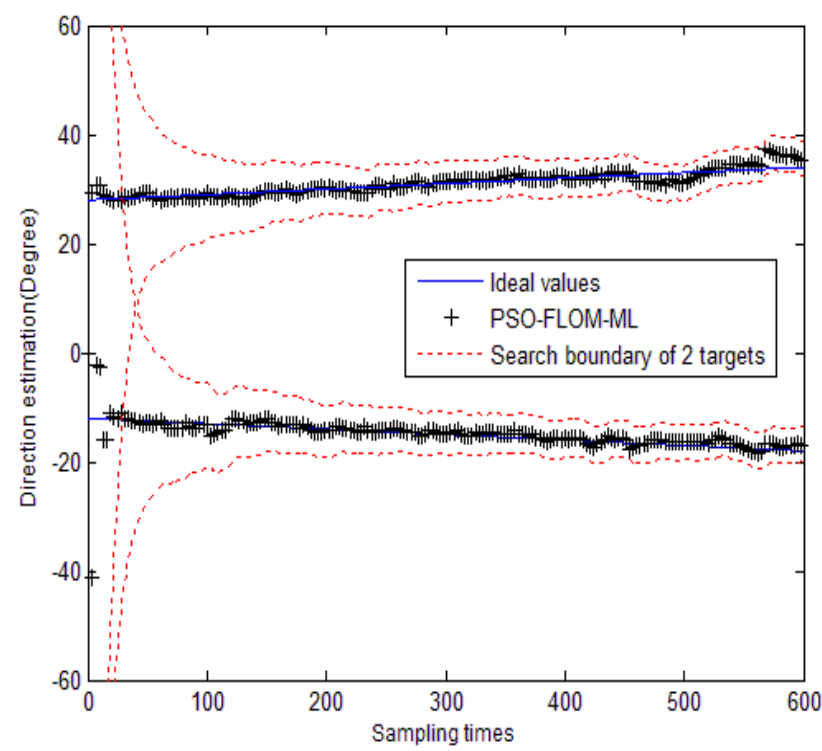

Figure 2. Tacking of two independent signal sources based on PSO-FLOM-ML

Similarly, three independent signal sources coming from $\theta_{1}(k)=[-30+5 \sin (2 \pi k / 500)]^{\circ}, \theta_{2}(k)=[13+5 \sin (2 \pi k / 500)]^{\circ}$ and $\theta_{3}(k)=[48+5 \sin (2 \pi k / 500)]^{\circ}$ are considered. Fig. 3 and Fig. 4 demonstrate the direction tracking performance of the two tracking algorithms for three independent signal sources with $\alpha=1.5$ and GSNR $=10 \mathrm{~dB}$. It can be seen that the tracking performance of QPSOWSC-ML is better than that of PSO-FLOM-ML.

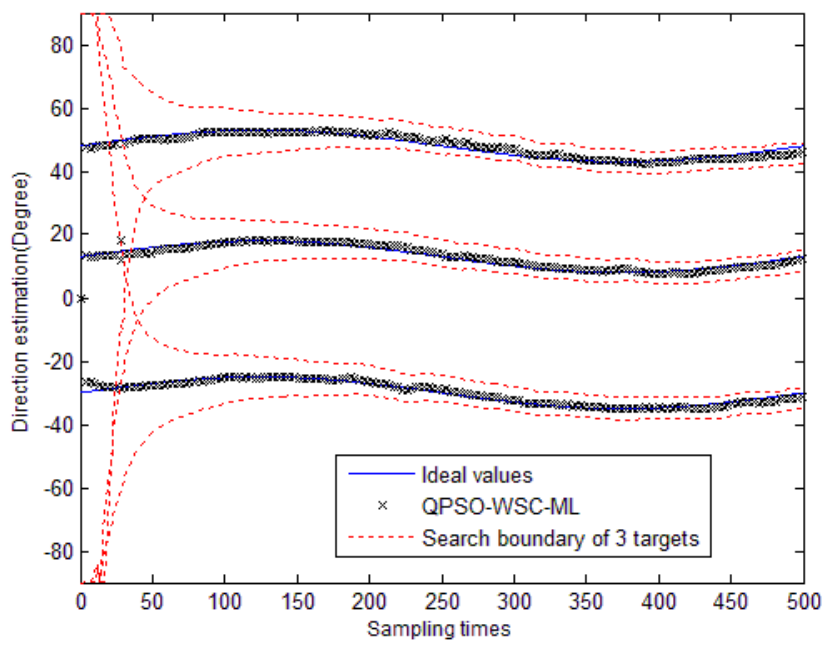

Figure 3. Tacking of three independent signal sources based on QPSO-WSC-ML

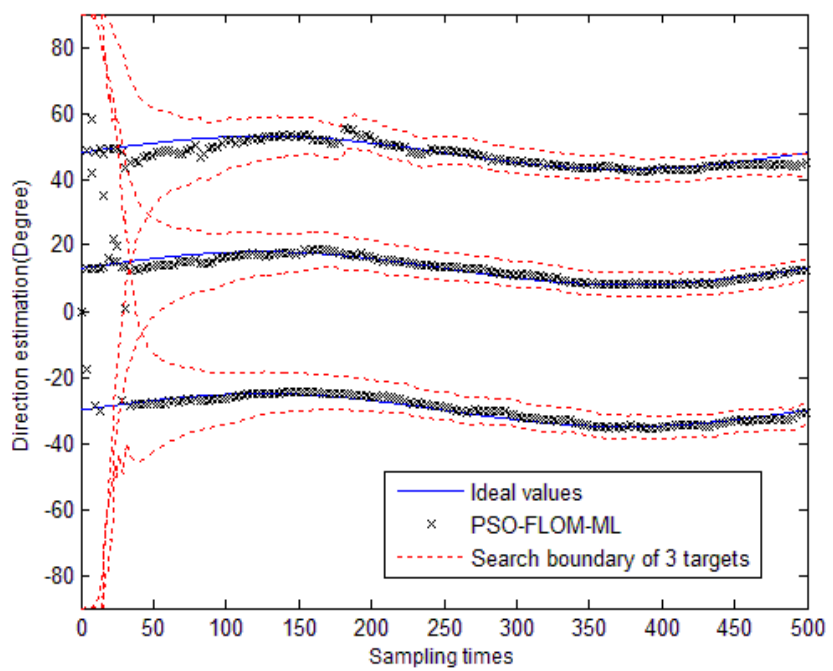

Figure 4. Tacking of three independent signal sources based on PSO-FLOM-ML

At last, Fig. 5 and Fig. 6 demonstrate the direction tracking performance of the two tracking algorithms for three coherent signal sources with $\alpha=1.5$ and $\mathrm{GSNR}=10 \mathrm{~dB}$. Compared with PSO-FLOM-ML, QPSOWSC-ML has more robust tracking performance. From Fig. 3-6, we can also see that it is easy to track independent signal sources with the same simulation condition. 


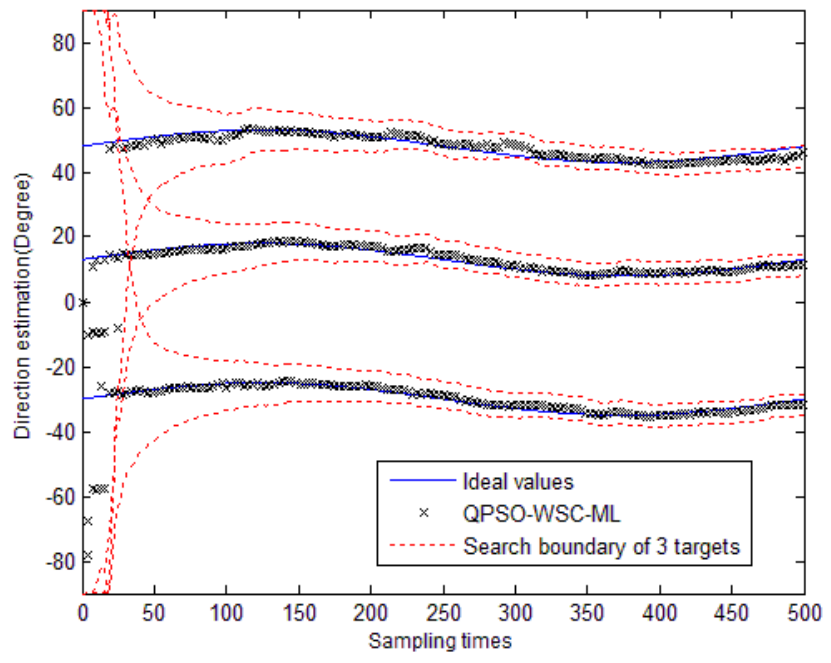

Figure 5. Tacking of three coherent signal sources based on QPSO-WSC-ML

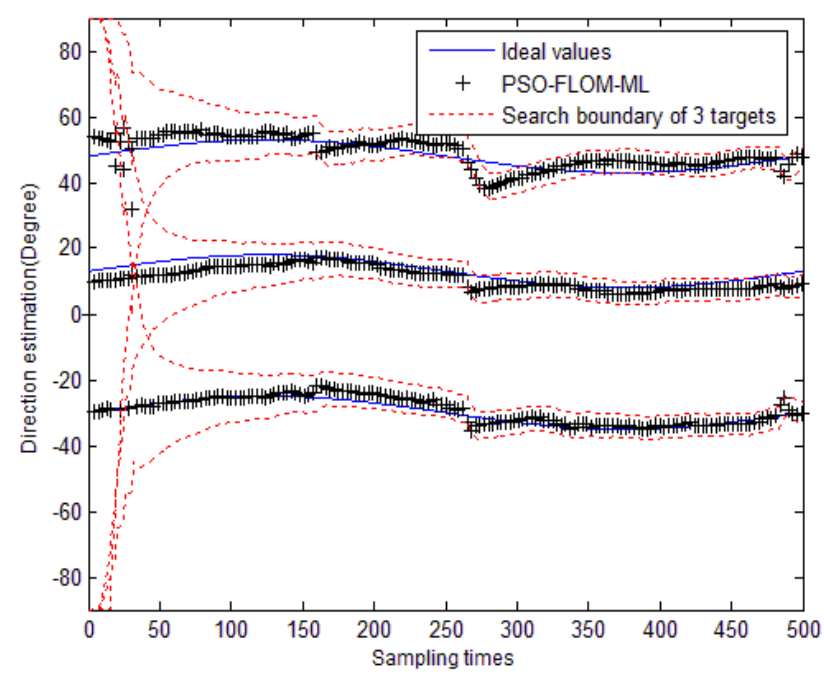

Figure 6. Tacking of three coherent signal sources based on PSO-FLOM-ML

\section{Conclusion}

Based on the WSC-ML and QPSO, we have proposed a novel method which is named as QPSO-WSC-ML for tracking multiple moving targets in the impulse noise environment. By QPSO for the continuous optimization problem of the direction tracking, we can reduce the computational cost of WSC-ML and improve the searching accuracy of previous PSO algorithm. Our future work is to use the proposed QPSO-WSC-ML method to MIMO radar, and we will try to convert the method into the impulsive noise environment.

\section{References}

1. H. Krim, and M. Viberg, IEEE Signal Processing Magazine, 13, 67-94 (1996)

2. X. Mestre, P. Vallet, P. Loubaton, Signal Processing Conference (EUSIPCO), 2013 Proceedings of the 21st European IEEE, 1-5 (2013)

3. J. Y. Lee, R. E. Hudson, K. Yao, IEEE Systems Journal, 8, 131-141 (2014)

4. Z. Y. He, Z. Q. Zhao, K. Yang, J. Ouyang, Computational Problem-Solving (ICCP), 2011 International Conference on IEEE, 405-408 (2011)

5. Y. L. Sit, M. Agatonovic, T. Zwick, Radar Conference (EuRAD), 2012 9th European, 298-301 (2012)

6. W. Shi, J. Huang, Y. S. Hou, Journal of Systems Engineering and Electronics, 23, 173-178 (2012)

7. K. V. S. Hari, V. Lalitha, Proc. IEEE International Conference on Acoustics, 7882, 2580-2583 (2011)

8. J. B. Long, D. F. Zha, H. B. Wang, International Conference on Mechatronic Sciences, Electric Engineering and Computer (MEC), 3504-3508 (2013)

9. H. Y. Gao, C. Q. Xu, International Journal of Computing Science \& Mathematics, 4, 321-331 (2013)

10. H. Y. Gao, C. W. Li, Acta Phys. Sin., 63, $128802-$ $130(2014)$

11. D. Y. Zhao, M. Diao, L. L. Yang, C. Chen, Journal of Shandong University, 40, 133-138 (2010) 